Check for updates

Cite this: RSC Adv., 2020, 10, 1042

Received 22nd October 2019

Accepted 9th November 2019

DOI: 10.1039/c9ra08651j

rsc.li/rsc-advances

\section{A fluorescence immunoassay based on CdTe : Zn/ ZnS quantum dots for the rapid detection of bacteria, taking Delftia tsuruhatensis CM'13 as an example $\uparrow$}

\begin{abstract}
Yao Ruan,,$^{\mathrm{a}}$ Huanhuan Xu, $\$^{\mathrm{b}}$ Jinlu Yu, ${ }^{\mathrm{b}}$ Qian Chen, ${ }^{\mathrm{a}}$ Lihong Gu${ }^{\mathrm{a}}$ and Ailing Guo (D) *ab
A fluorescence immunoassay has been widely applied in different fields due to its high sensitivity, simple operations, and high accuracy. Quantum dots (QDs) are often selected as labels in a fluorescence immunoassay due to their high fluorescence, better stability, and biocompatibility. In this study, novel doped CdTe : Zn/ZnS QDs with stability and a high photoluminescence quantum yield (40.78\%) were prepared by the water synthesis method and used as labels to conjugate with goat anti-rabbit $\lg G$ to establish a fluorescence immunoassay (FLISA) for bacteria compared to the traditional enzyme-linked immunosorbent assay (ELISA) based on the reaction between an antibody and an antigen. A good linear relationship between the fluorescence intensity and concentrations of $D$. tsuruhatensis CM13 was found when the concentrations were in the range of $10^{3} \mathrm{CFU} \mathrm{mL} \mathrm{L}^{-1}-10^{8} \mathrm{CFU} \mathrm{mL} \mathrm{m}^{-1}$. The limit of detection (LOD) of $D$. tsuruhatensis CM13 was $1.25 \times 10^{3} \mathrm{CFU} \mathrm{mL}{ }^{-1}$ by FLISA, which was about 80 times lower than the LOD obtained from ELISA $\left(10^{5} \mathrm{CFU} \mathrm{mL}{ }^{-1}\right)$. This indicated that our FLISA method has higher sensitivity than traditional ELISA, and the CdTe:Zn/ZnS QDs synthesized in this paper have good applications in the rapid sensitive detection of microorganisms.
\end{abstract}

\section{Introduction}

Quantum dots (QDs) are nanocrystals made of semiconductor materials often composed of atoms from group II-VI or III-V elements in the periodic table and are defined as particles with physical dimensions smaller than the exciton Bohr radius (they have sizes in the range of 1-10 nm). Based on the quantum confinement effect, the electrons and holes in the interior of QDs are locked in a tiny three-dimensional space, so that the continuous energy band structure is converted into a discrete structure having molecular activity to emit fluorescence. QDs possess many unique and distinctive characteristics. For example, the emission spectrum can be adjusted by changing the size of QDs; ${ }^{1}$ the stability of fluorescence is good and the fluorescence intensity is 20 times that of rhodamine. ${ }^{2}$ Moreover, QDs exhibit wide excitation spectra and narrow emission spectra, due to which they can be used to achieve simultaneous detection; QDs also have good biocompatibility. They can be combined with

${ }^{a}$ National Research and Development Center for Egg Processing, Huazhong Agriculture University, Wuhan 430070, Hubei Province, China. E-mail: 610638206@qq.com; 631185610@qq.com; 384023595@qq.com; ailingguo234@163.com; Tel: +8615342241896 ${ }^{b}$ Key Laboratory of Environment Correlative Dietology (Huazhong Agricultural University), Ministry of Education, Wuhan 430070, China.E-mail: 2440289275@qq. com; jinluyu@webmail.hzau.edu.cn

$\dagger$ Electronic supplementary information (ESI) available. See DOI: 10.1039/c9ra08651j

\$ Yao Ruan and Huanhuan Xu equally contributed to this study. some biomolecules to act as markers to detect living organisms. ${ }^{3-5}$ At present, QDs have received extensive attention in the biological, chemical, and optoelectronic fields. ${ }^{6-8}$

Cd-based QDs have become a hot research topic due to their higher fluorescence quantum yields. ${ }^{\mathbf{9}, 10}$ However, due to the disadvantages of core QDs (CdTe), such as aggregation, poor fluorescence stability, short shelf life, and easily lost fluorescence, ${ }^{1}$ they can be used on newly prepared materials briefly. A single-core QD nanomaterial is coated with other compounds to form core-shell QD. The presence of the shell reduces the surface defects and protects the core from decomposition under photoexcitation, thereby increasing the fluorescence yield, fluorescence intensity, and stability of mononuclear quantum dots. ${ }^{11}$ With the development of nanomaterials by researchers, a new type of doped QDs could be synthesized. ${ }^{12}$ Doped QDs refer to nanocrystals formed by adding a small amount of transition metal ions or rare earth ions during the synthesis of QDs. The excitation wavelength of the synthesized doped QDs depends mainly on the band gap of the host material and the wavelength of the luminescence is related to the doped atoms selected. The advantage of doping QDs is that they have a large Stokes displacement, which helps prevent the phenomenon of self-priming. Therefore, CdTe : Zn/ZnS-doped QDs synthesized in this study have a longer fluorescence lifetime, stronger photostability, and higher fluorescence efficiency compared with traditional CdTe/ZnS QDs, which indicates that doped QDs are attractive fluorescent probes for use in the analytical detection field. 
Fluorescent immunoassay (FLISA) is an efficient highthroughput detection method. The principles of its operation and detection are similar to ELISA. The difference between ELISA and FLISA is that ELISA uses enzyme-labeled secondary antibodies to determine the absorbance of the substrate, while FLISA employs a fluorescent substance as the label to measure the fluorescence intensity. The QDs-based FLISA is being widely studied nowadays. Zhang et al. used functionalized Cd-doped ZnO QDs to covalently couple with bisphenol A (BPA) antibody to detect BPA by competitive FLISA. The effective linear working range was $20.8-330.3 \mathrm{ng} \mathrm{mL}{ }^{-1}$ and the LOD was $13.1 \mathrm{ng} \mathrm{mL}{ }^{-1} .^{13}$ Xiong et al. reported a new direct competition dcFLISA for the detection of ochratoxin A (OTA) by introducing large size polymer bead-loaded quantum dots (QBs). At optimal parameters, the linear range of dcFLISA for OTA detection was $0.05 \mathrm{pg} \mathrm{mL}^{-1}-1.56$ $\mathrm{pg} \mathrm{mL^{-1 }}$ with a maximum inhibitory concentration of $0.14 \pm 0.04$ pg $\mathrm{mL}^{-1}(n=5) .{ }^{14}$ Yang et al. introduced FLISA for specific quantitative detection of bovine alpha-lactalbumin ( $\alpha$-La) in dairy products. It was found that there was a good linear relationship between the inhibition efficiency and the logarithm of $\alpha$-La's concentrations after optimization. The $\alpha$-La's concentrations ranged from $0.1-1000 \mathrm{ng} \mathrm{mL}^{-1}$, the half maximal inhibitory concentration $\left(\mathrm{IC}_{50}\right.$ ) was $0.03 \mu \mathrm{g} \mathrm{mL}{ }^{-1}$, and LOD was $0.1 \mathrm{ng}$ $\mathrm{mL}^{-1}$, showing that the established FLISA had sensitivity and was successfully applied to the detection of $\alpha$-La in commercial dairy products. ${ }^{15}$ There are also other methods using QDs such as impedance, ${ }^{16}$ ELISA, ${ }^{17}$ test strips $^{18,19}$ and PCR for detecting bacteria. ${ }^{20,21}$ Above all, FLISA has been widely used for detection in many fields, while the application of FLISA in microbiological detection is correspondingly rare.

In this study, we successfully established FLISA to detect $D$. tsuruhatensis CM13 with the help of the high fluorescence, good stability, and good biocompatibility of QDs. Compared with the traditional ELISA (Fig. 1), our new FLISA provided rapid and accurate detection method of food spoilage bacteria, ensuring the quality and safety of food during storage and transportation.

\section{Experiment}

\section{Materials and reagents}

The D. tsuruhatensis CM13 used in the experiments were isolated and purified from eggs purchased from the Jiufengshan breeding site. Other strains were provided by Center for Disease Control and Prevention of HuBei, WuHan. Sodium borohydride $\left(\mathrm{NaHB}_{4}\right)$ and cadmium chloride $\left(\mathrm{CdCl}_{2}\right)$ were purchased from Sinopharm Chemical Reagent Co., Ltd; 1-(3-dimethylaminopropyl)-3ethylcarbodiimide hydrochloride $(\mathrm{EDC} \cdot \mathrm{HCl})$ and $N$-hydroxysuccinimide (NHS) were obtained from Shanghai Crystal Pure Reagent Co., Ltd. Goat anti-rabbit IgG-HRP and Goat anti-rabbit IgG were provided by Hubei Jing Mao Biotech Co., Ltd and Boster Biological Technology Co., Ltd, respectively.

\section{Preparation of water-soluble CdTe : Zn/ZnS QDs}

One hundred $\mathrm{mg} \mathrm{NaHB}_{4}$ was quickly added into the reaction flask wrapped with aluminum membrane with $3 \mathrm{~mL}$ of ultrapure water and $63.8 \mathrm{mg}$ Te powder with the needle inserted on the lid to discharge hydrogen, then put in a water bath at $40{ }^{\circ} \mathrm{C}$ for $1 \mathrm{~h}$ until the solution was clear purple with white precipitate to obtain sodium hydrogen telluride precursor.

After a series of optimization (ESI $1 \dagger$ ), we took $91.34 \mathrm{mg}$ of $\mathrm{CdCl}_{2}$ and $43.9 \mathrm{mg}$ of zinc acetate, and dissolved them in 100.0 mL ultrapure water. Then, $78 \mu \mathrm{L}$ of MPA was added subsequently and the $\mathrm{pH}$ value was adjusted to 7.5 by $0.1 \mathrm{~mol} \mathrm{~L}^{-1}$ sodium hydroxide. After aerating with nitrogen under magnetic stirring for $30 \mathrm{~min}$, sodium hydrogen telluride precursor was added and heated at $100{ }^{\circ} \mathrm{C}$ for $2.5 \mathrm{~h}$ to prepare CdTe : Zn QDs.

Fifty $\mathrm{mL}$ CdTe : Zn QDs solution in a four-neck flask was filled with nitrogen. After a series of optimization (ESI $2 \dagger$ ), we decided to magnetically stir for $30 \mathrm{~min}$ and heat at $85^{\circ} \mathrm{C}$, then quickly added $0.5 \mathrm{~mL}$ zinc acetate solution $\left(0.1 \mathrm{~mol} \mathrm{~L}^{-1}\right)$ and $0.5 \mathrm{~mL}$ of sulfur the acetamide solution $\left(0.1 \mathrm{~mol} \mathrm{~L}^{-1}\right)$, finally refluxing for $3.5 \mathrm{~h}$ to obtain CdTe : $\mathrm{Zn} / \mathrm{ZnS}$ QDs.

\section{Preparation of $D$. tsuruhatensis CM13 polyclonal antibody}

D. tsuruhatensis CM13 polyclonal antibody was prepared by immunizing New Zealand white rabbits by multiple-site subcutaneous injection, using heat-inactivated $D$. tsuruhatensis CM13 with initial immune dose of $10^{7} \mathrm{CFU} \mathrm{mL}^{-1}(1$ $\mathrm{mL})$, immunizing the rabbits with a dose of $10^{8} \mathrm{CFU} \mathrm{mL} \mathrm{m}^{-1}(1$ $\mathrm{mL}$ ) every 2 weeks for three months, and collecting the blood from the ear vein on the seventh day after each immunization to measure titers by indirect ELISA. The titer of $D$. tsuruhatensis CM13 polyclonal antibody was 1 : 12800 after purification and the molecular weight was $148 \mathrm{kD}$ by sodium dodecyl sulfate polyacrylamide gel electrophoresis. Our animal experiment was approved by Huazhong Agricultural University and carried out in strict compliance with the guidelines of China Experimental Animal Welfare Ethics Committee.

\section{Conjugation of CdTe : Zn/ZnS QDs and antibody}

Purification of CdTe : Zn/ZnS QDs. With magnetic stirring, the ethanol solution was slowly added to the prepared CdTe : Zn/ZnS QDs solution until the turbidity appeared, continuing to stir for $5 \mathrm{~min}$, and centrifuged at $5000 \mathrm{rpm}$ for $5 \mathrm{~min}$. The supernatant was removed and the procedure was repeated three times. Then, the precipitate was re-dissolved with ultrapure water and stored under dark.

We employed amino acid-thiol covalent method to conjugate CdTe : Zn/ZnS QDs with antibody after optimizing (ESI $3 \dagger$ ). $120 \mu \mathrm{L}$ of CdTe : Zn/ZnS QDs solution was mixed with $\mathrm{EDC} \cdot \mathrm{HCl}(80 \mu \mathrm{L}$, $\left.0.05 \mathrm{~mol} \mathrm{~L}^{-1}\right)$ and NHs $\left(60 \mu \mathrm{L}, 0.05 \mathrm{~mol} \mathrm{~L}^{-1}\right)$, and then, $720 \mu \mathrm{L}$ of MES buffer ( $\mathrm{pH}$ 5.5) was added. After standing for $30 \mathrm{~min}$ avoiding light at room temperature and centrifuging the mixed solution at $12000 \mathrm{rpm}$ for $2 \mathrm{~min}$, the precipitate was re-dissolved in borate buffer ( $\mathrm{pH}$ 9.0). Then, $120 \mu \mathrm{L}$ of the antibody $\left(1 \mathrm{mg} \mathrm{mL}^{-1}\right)$ was added and reacted at $37{ }^{\circ} \mathrm{C}$ in a shaker for $2 \mathrm{~h}$ under dark. The mixed solution was transferred to $4{ }^{\circ} \mathrm{C}$ overnight. In the following day, centrifugation of the solution at $12000 \mathrm{rpm}$ for $30 \mathrm{~min}$ was done and the precipitates were stored in PBS buffer $\left(0.01 \mathrm{~mol} \mathrm{~L}^{-1}\right.$, $\mathrm{pH}$ 7.4) at $4{ }^{\circ} \mathrm{C}$ in the dark. 

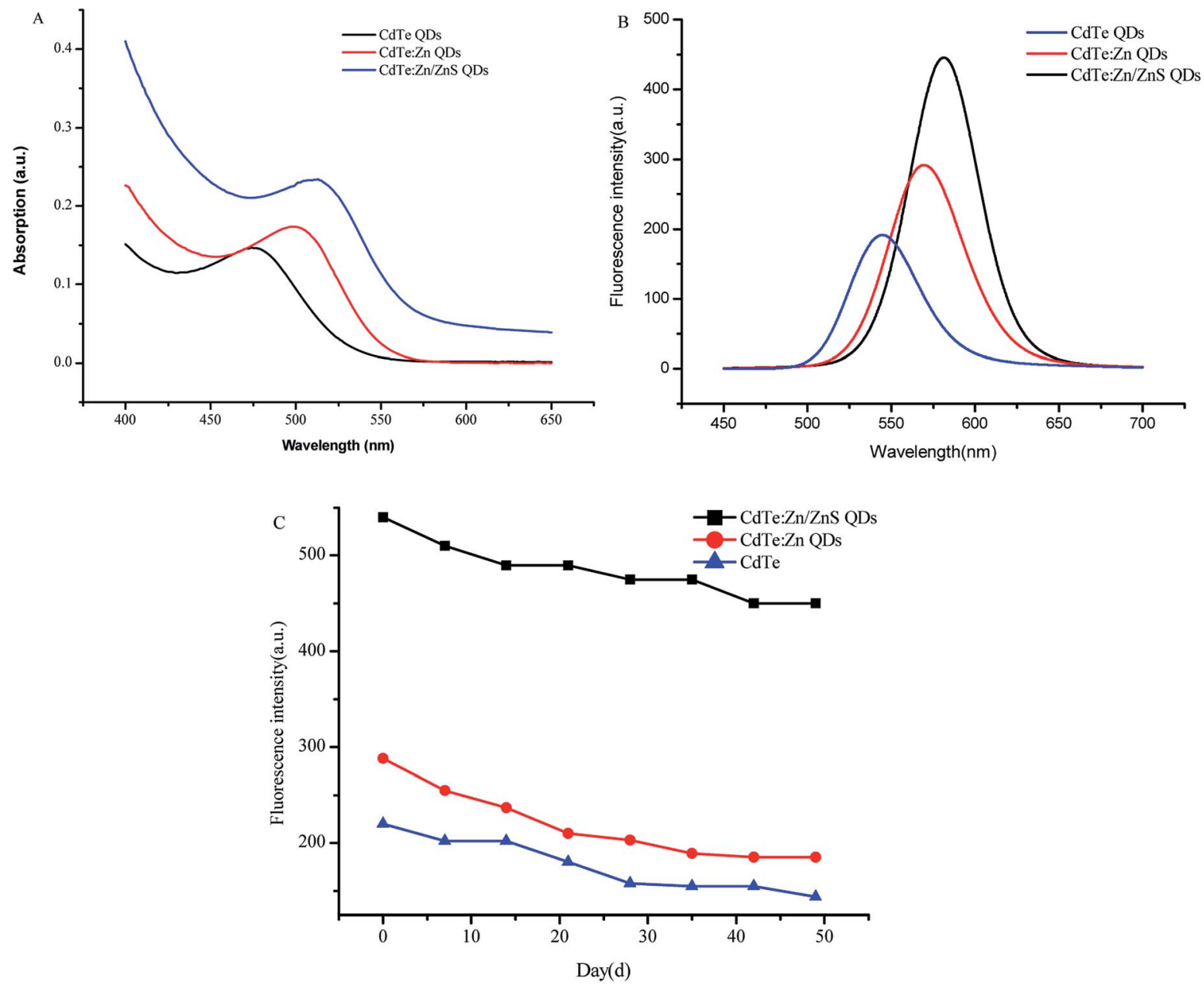

Fig. 1 The absorption spectrum (A) of CdTe, CdTe : Zn, and CdTe : Zn/ZnS QDs was obtained from $400 \mathrm{~nm}$ to $650 \mathrm{~nm}$ and the fluorescence emission spectrum (B) was tested in the range of $450 \mathrm{~nm}$ to $700 \mathrm{~nm}$. (C) Comparisons of stability between CdTe : Zn/ZnS QDs, CdTe : Zn QDs, and CdTe QDs.

\section{Established of FLISA for the detection of $D$. tsuruhatensis CM13}

The operation of FLISA was similar to ELISA. Firstly, a certain concentration of antigen $\left(10^{8} \mathrm{CFU} \mathrm{mL} \mathrm{m}^{-1}\right)$ was centrifuged and washed three times. Secondly, the blocking solution (10\% BSA, $200 \mu \mathrm{L}$ ) was added at $37^{\circ} \mathrm{C}$ for $1.5 \mathrm{~h}$, centrifuged, and washed 3 times. Then, rabbit serum was added and incubated at $37^{\circ} \mathrm{C}$ for $1 \mathrm{~h}$, centrifuged, and washed 3 times. All of the above conditions have been properly optimized (ESI $4 \dagger$ ). After that,
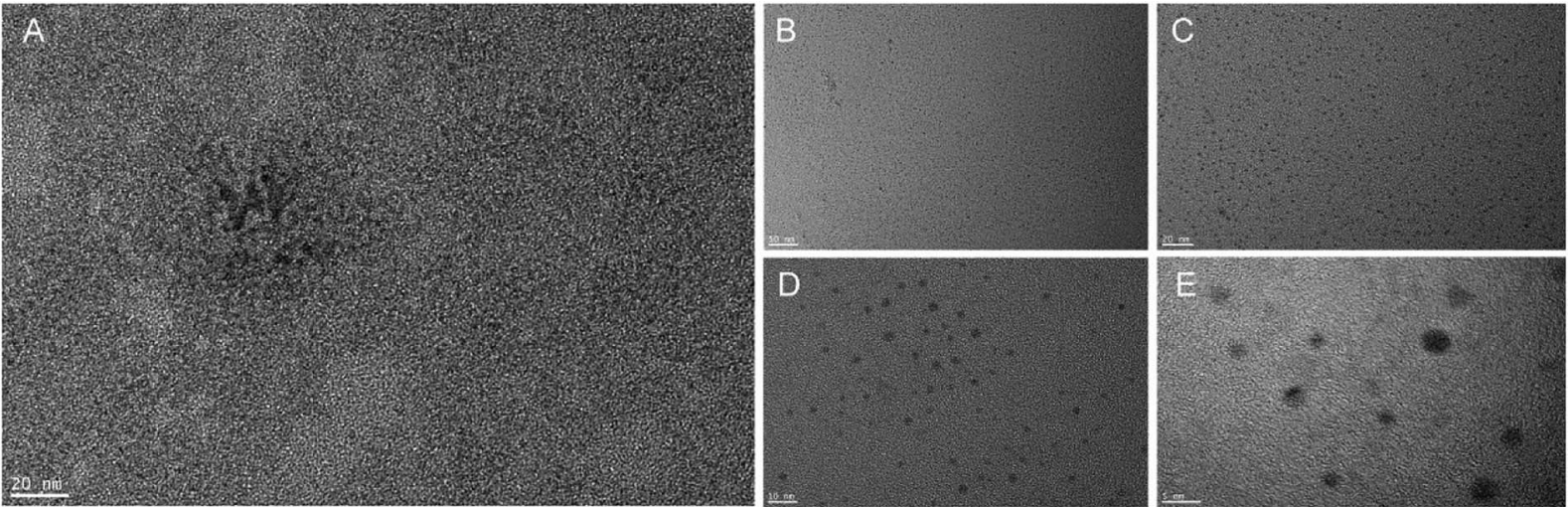

Fig. 2 TEMs of CdTe : Zn QDs (A) and CdTe : Zn/ZnS QDs (B-E). Note: (B-E) were CdTe : Zn/ZnS QDs under 50 nm, 20 nm, 10 nm, 5 nm field of vision, respectively. 


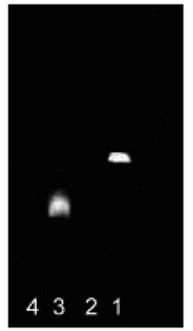

Fig. 3 Agarose gel fluorescent spectra of CdTe : Zn/ZnS QDs before and after conjugating with antibody. Note: lane 1-CdTe : Zn/ZnS QDs; lane 2-antibody; lane 3-CdTe : Zn/ZnS QDs-antibody, lane 4-PBS.

CdTe : Zn/ZnS QDs-labeled goat anti-rabbit IgG was added and incubated at $37{ }^{\circ} \mathrm{C}$ for $1 \mathrm{~h}$, centrifuged, and washed 5 times. Finally, the precipitates were dissolved in PBS and transferred to a black clear bottom 96-well plate measured by a multifunctional microplate reader. The excitation wavelength was $528 \mathrm{~nm}$. The measurement method was bottom reading.

\section{Results and discussion}

\section{Characterization of the QDs}

The fluorescence emission spectra and UV absorption spectra of the QDs are shown in Fig. 1. From the emission spectra of QDs, the fluorescence emission peaks were about 545, 565, and $578 \mathrm{~nm}$, respectively. The fluorescence intensity of CdTe : $\mathrm{Zn} / \mathrm{ZnS}$ QDs is $140 \%$ higher than that of CdTe QDs. The full wavelength at half maximum (FWHM) of the emission spectrum was about $45 \mathrm{~nm}$, which indicated that the prepared CdTe : Zn/ZnS QDs solution had better chromatic dispersion. In addition, the UV absorption spectra showed that the maximal absorption of CdTe was approximately $475 \mathrm{~nm}$. The CdTe : $\mathrm{Zn}$ and CdTe : Zn/ZnS QDs had shifted their peaks at $500 \mathrm{~nm}$ and $525 \mathrm{~nm}$, respectively. The stability of the QDs is shown in Fig. 1C, which have a strong fluorescence signal. Additionally, Fig. 2 shows the transmission electron microscopic images of the QDs. It could be seen that CdTe and CdTe: Zn QDs were aggregated easily. However, CdTe : Zn/ZnS QDs were composed of linear crystals with less agglomeration, uniform distribution, and a particle size of approximately $3 \mathrm{~nm}$.

The characterization results demonstrated that the prepared core-shell QDs doped Zn has good spectral properties and aqueous dispersion that can be utilized as fluorescent probes in bioanalysis.

\section{Conjugation of CdTe : Zn/ZnS QDs and antibody}

The conjugation of the antibody and CdTe : Zn/ZnS QDs was observed by agarose gel electrophoresis. The results are shown in Fig. 3. Lanes 1-4 were added corresponding to CdTe : Zn/ZnS QDs, antibody, CdTe : Zn/ZnS QDs-antibody, and PBS, respectively. From Fig. 3, we could find that the CdTe : Zn/ZnS QDs were conjugated with the antibody because of slower migration with increased molecular weight. The emission spectrum was also detected. Compared to QDs, the antibody-conjugated product shows $27.65 \%$ lower fluorescence intensity in Fig. 4 .

\section{Activity of antibody after coupling with CdTe : Zn/ZnS QDs}

The activity of the antibody coupled to CdTe : Zn/ZnS QDs was determined by dot blotting. $1 \mu \mathrm{L}, 5 \mu \mathrm{L}, 10 \mu \mathrm{L}$, and $15 \mu \mathrm{L}$ of antigen solution $\left(10^{7} \mathrm{CFU} \mathrm{mL} \mathrm{m}^{-1}\right)$ were added to the nitrocellulose membrane (b-e) and the PBS buffer was the blank control (a). The results are shown in Fig. 5 . It could be seen that more the amount of antigen added, the higher the brightness of the spot under UV imaging. On the other hand, the weaker light in the blank area was caused by non-specific adsorption. It could be inferred that the antibody was successfully coupled to CdTe : Zn/ZnS QDs and the antibody still retained its biological activity after being conjugated with CdTe : Zn/ZnS QDs (Fig. 6).

\section{Detection of $\boldsymbol{D}$. tsuruhatensis CM13 by ELISA and FLISA}

The FLISA method is a kind of detection method similar to the ELISA method; they have similar detection principles and
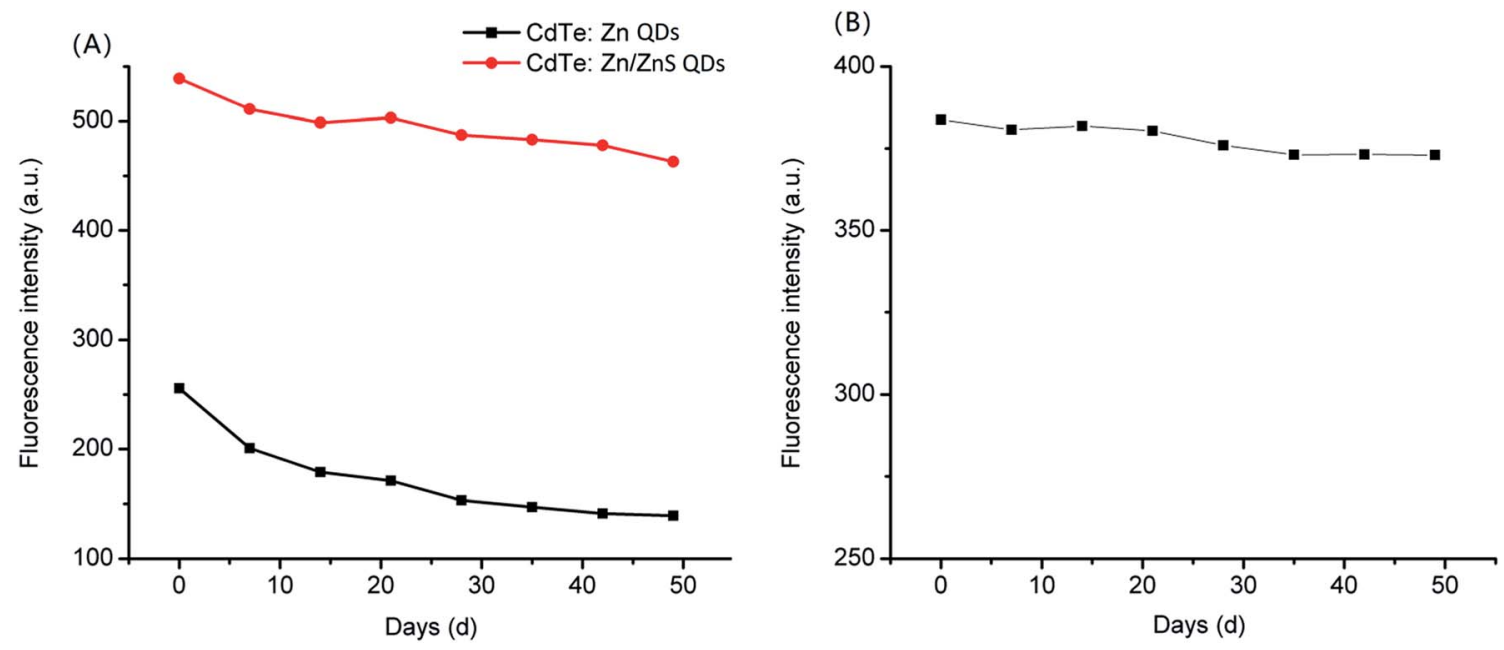

Fig. 4 Stability of CdTe : Zn/ZnS QDs and CdTe : Zn QDs (A); stability of CdTe : Zn/ZnS QDs-antibody (B). 


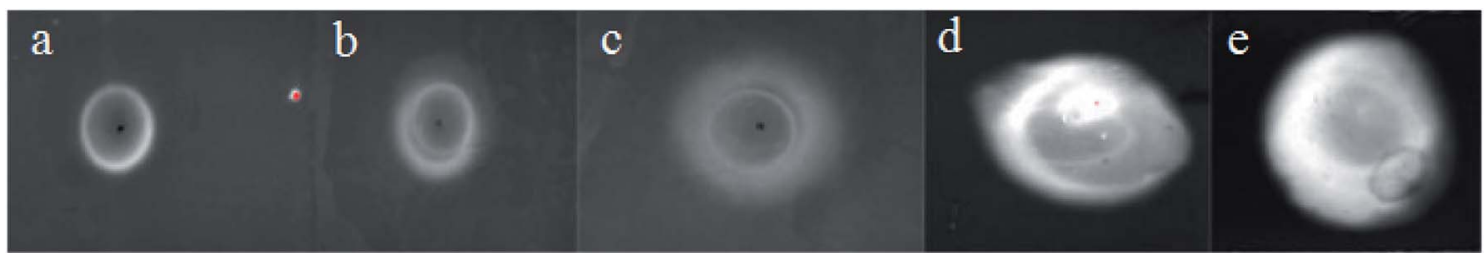

Fig. 5 The ultraviolet imagery of spots mark. Note: (a) was PBS; (b)-(e) were expressing the amount of antigen were $1 \mu \mathrm{L}, 5 \mu \mathrm{L}, 10 \mu \mathrm{L}$, and $15 \mu \mathrm{L}$, respectively.

operation procedures. The difference between the FLISA method and the ELISA method is that the FLISA method eliminates the step of coloration of the coated antigen and the substrate, which greatly saves time. In addition, the ELISA method is based on the OPD color reaction, the OD value at $492 \mathrm{~nm}$ to reflect the amount of the antigen, FLISA is used to measure the antigen by measuring the fluorescence intensity of the CdTe : Zn/ZnS QDs-labeled goat anti-rabbit IgG-polyclonal antibody-antigen.

In this study, D. tsuruhatensis CM13 was detected by ELISA and FLISA in Fig. 7, respectively. The LOD by ELISA and FLISA were $5 \log \mathrm{CFU} \mathrm{mL} \mathrm{mL}^{-1}$ (with the linear correlation of $10^{5} \mathrm{CFU}$ $\mathrm{mL}^{-1}-10^{8} \mathrm{CFU} \mathrm{mL} \mathrm{m}^{-1}$ ) and $3.097 \log \mathrm{CFU} \mathrm{mL} \mathrm{mL}^{-1}$ (with the linear correlation of $10^{3} \mathrm{CFU} \mathrm{mL} \mathrm{mL}^{-1}-10^{8} \mathrm{CFU} \mathrm{mL}^{-1}$ ), respectively. The FLISA method was 80 times more sensitive than the ELISA.

\section{Artificial simulation experiments}

In artificial simulation experiments, low-fat milk was diluted 10-fold. With a series of concentrations of D. tsuruhatensis CM13 mixed, it was detected by ELISA and FLISA in Fig. 8, respectively. In this experiment, LOD of D. tsuruhatensis of FLISA (4.398 $\log$ CFU mL ${ }^{-1}$ ) was about 40 times more sensitive than ELISA ( $6 \log$ CFU mL $\left.{ }^{-1}\right)$.

\section{Stability of the FLISA method}

In the FLISA method, the stability of FLISA was decided by CdTe : Zn/ZnS QDs-labeled goat anti-rabbit IgG. When storing for $74 \mathrm{~d}$, the conjugates showed more severe sedimentation, which may be due to the agglomeration of CdTe : Zn/ZnS QDsantibody conjugates after long-term standing. When storing for 102 d, the CdTe : Zn/ZnS QDs CdTe : Zn/ZnS QDs-labeled goat anti-rabbit IgG became dark and the fluorescence disappeared. This may be due to the mix of oxygen at the same time that the CdTe : Zn/ZnS QDs-antibody conjugates with the agglomerate, which oxidized the CdTe : Zn/ZnS QDs and became dark; thus, the fluorescence disappeared.

D. tsuruhatensis CM13 was detected by FLISA every week. As shown in Table 1, when the detection reagents of FLISA method were stored for $35 \mathrm{~d}$, the LOD was changed to $3.699 \log \mathrm{CFU}$ $\mathrm{mL}^{-1}$. When storing for $81 \mathrm{~d}$, the LOD was changed to $4 \log \mathrm{CFU}$ $\mathrm{mL}^{-1}$. If stored for $88 \mathrm{~d}$, the LOD was $5.989 \log \mathrm{CFU} \mathrm{mL}{ }^{-1}$. It could be found that with the extension of storage time, the LOD of D. tsuruhatensis CM13 was reduced.

\section{Comparison of the FLISA method with other detection methods}

At present, metabolic methods, molecular biology methods, and immunological methods are popular for detecting microorganisms. From Table 2, the LOD of ELISA, one of the typical
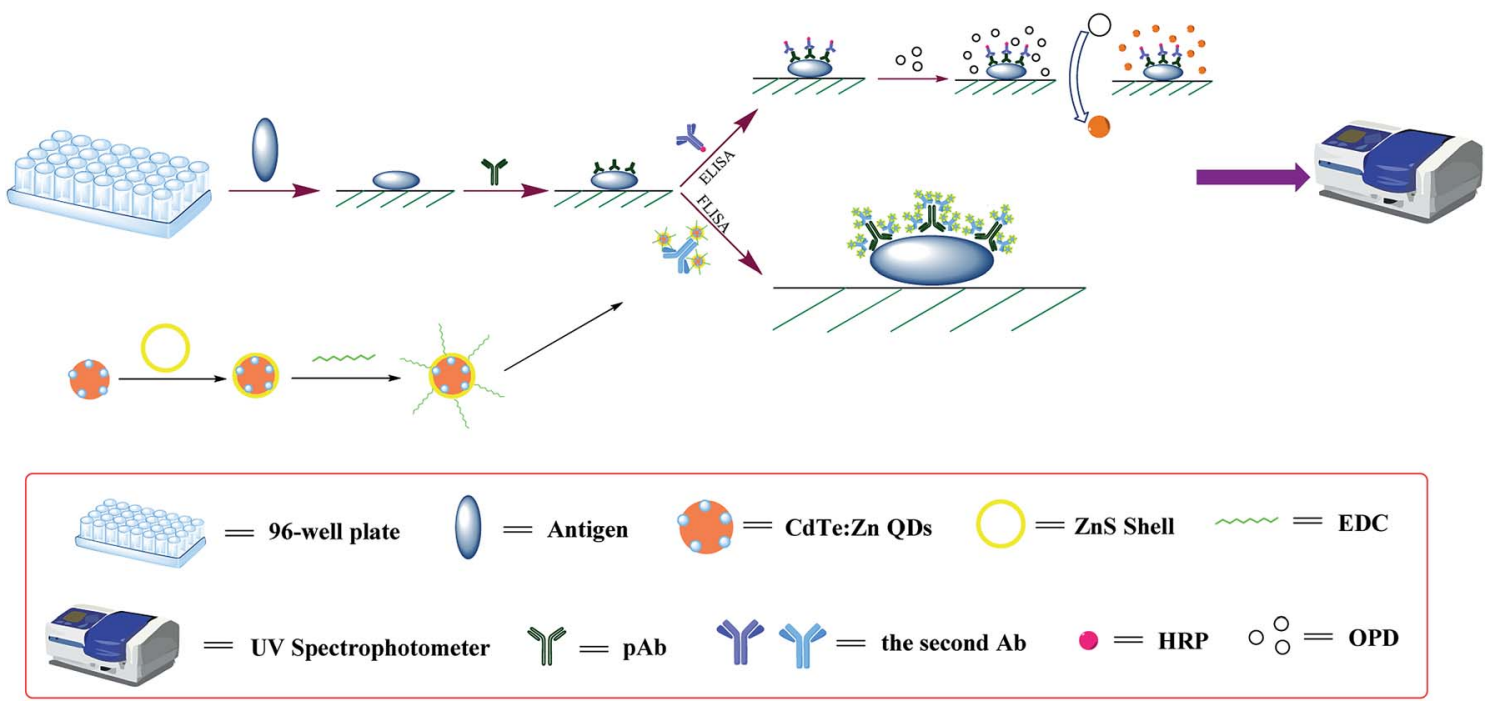

Fig. 6 A schematic diagram of detection principles of ELISA and FLISA, respectively. 

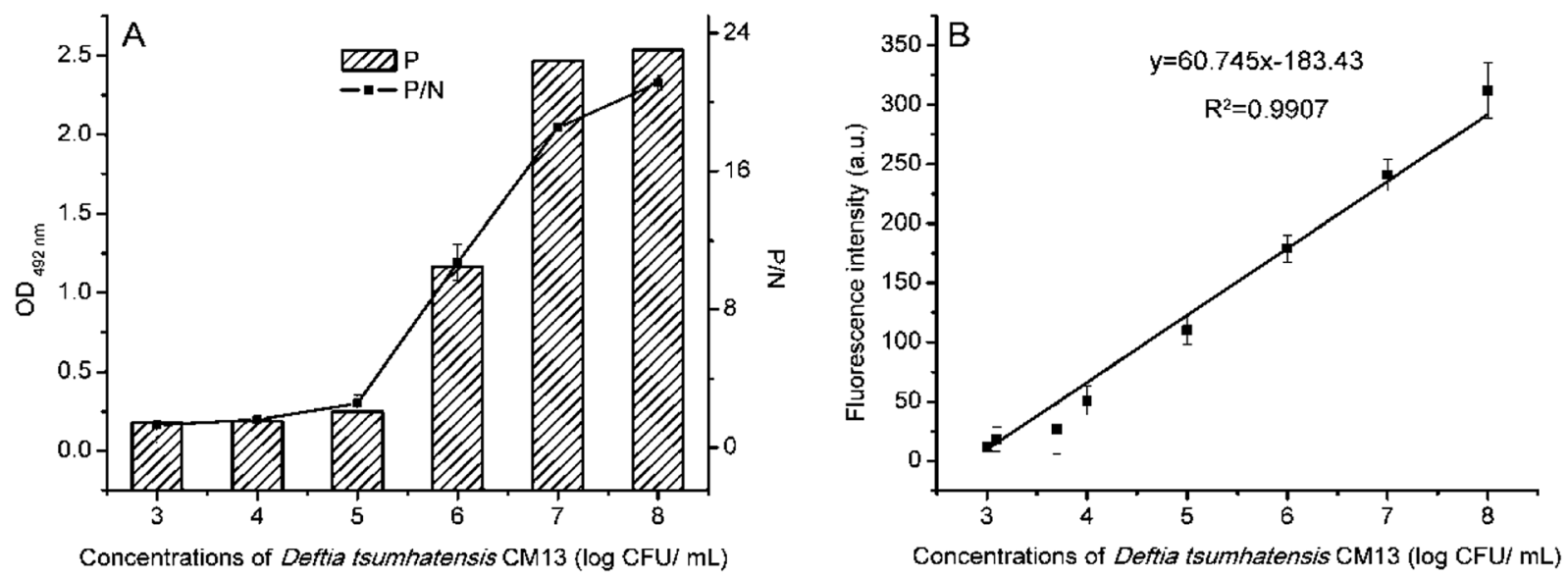

Fig. 7 Detection limits of ELISA (A) and FLISA (B) for detecting D. tsuruhatensis CM13, respectively.

immunological method, is about $10^{3}-10^{6} \mathrm{CFU} \mathrm{mL}^{-1}$. In addition, our study used also ELISA to detect $D$. tsuruhatensis CM13 and the LOD was $10^{5} \mathrm{CFU} \mathrm{mL}^{-1}$. In real detection, the microorganisms just contaminate most food at low levels, while the lower LOD of ELISA cannot detect microorganisms directly without enrichment. It may result in negative results or excessive detection time and limit wide application in food companies and actual testing. The immunochromatographic test strip is one of the immunoassay methods. The most widely used is colloidal gold immunochromatographic test strips. The LOD is generally $10^{5}-10^{6} \mathrm{CFU} \mathrm{mL}{ }^{-1}$. However, with the development of immunochromatographic test strips, various fluorescent substances (such as quantum dots) have also appeared as markers to detect microorganisms, so that the detection limit is 10-100 times higher than that of colloidal gold (up to $10^{4} \mathrm{CFU}$ $\left.\mathrm{mL}^{-1}\right){ }^{5}$ On the other hand, the fluorescence of QDs will reduce or disappear due to oxidation during storage, leading to the poor stability of the test strips (effect 30 days only). PCR and metabolic methods have both lower LOD, especially PCR, where the LOD of the microorganisms can be up to $10^{2} \mathrm{CFU} \mathrm{mL}^{-1}$ but the process of PCR to detect microorganisms, such as increasing bacteria, extracting DNA, amplification, and electrophoresis, are necessary for low bacterial concentration. Also, the operation is complicated. Metabolic methods are usually used to determine the total number of microorganisms. ATP method, which is one of the metabolic methods, is relatively mature and widely used. It also has good sensitivity in detecting the total number of microorganisms, while the ATP from different types of microorganisms is same, so the result is sometimes not very accurate. An impedimetric biosensing technique, which is an electrochemical method, offers several advantages such as good sensitivity, miniaturization potential, and mass production. ${ }^{23,24}$ However, this technology is costly and technically strong, and its application is difficult to popularize. In this study, we successfully established a FLISA method to detect $D$. tsuruhatensis CM13; the LOD was about $1.25 \times 10^{3}$ CFU $\mathrm{mL}^{-1}$, which was about 80 times more sensitive than the ELISA method. In addition, the FLISA method was simple, had a short detection time (about $4 \mathrm{~h}$ ), low cost, high sensitivity, and could be used to directly detect food. But some samples with $15 \%$ salt ( $\mathrm{NaCl}$ ), $10 \%$ sugar (glucose), $8 \%$ amino acid (L-Met), or $6 \%$ protein (whey protein), the detection must be performed
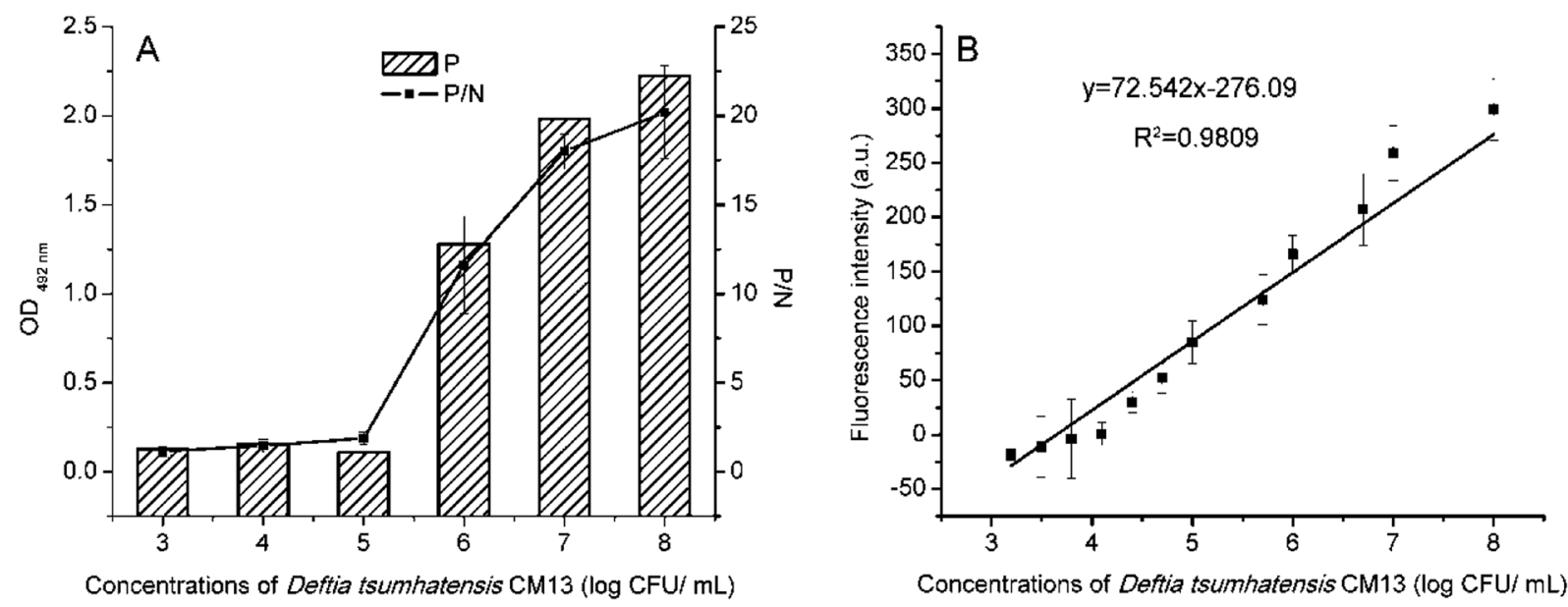

Fig. 8 Detection limits of ELISA (A) and FLISA (B) for detecting D. tsuruhatensis CM13 in artificial contamination test. 
Table 1 Stability of FLISA for the detection of D. tsumhatensis CM13tab1fna

\begin{tabular}{|c|c|c|c|c|c|c|c|c|c|c|c|c|c|c|c|}
\hline \multirow[b]{2}{*}{ Fluorescence intensity (a.u.) } & & \multicolumn{14}{|c|}{ Day } \\
\hline & & 0 & 7 & 14 & 21 & 28 & 35 & 42 & 49 & 59 & 66 & 74 & 81 & 88 & 95 \\
\hline & 4 & 53 & 51 & 44 & 34 & 27 & 28 & 34 & 35 & 30 & 35 & 12 & 22 & 16 & - \\
\hline & 3.699 & 21 & 29 & 29 & 14 & 14 & 11 & 8 & 13 & 10 & 7 & 4 & 4 & - & - \\
\hline & 3.098 & 12 & 11 & 5 & 3 & 3 & 7 & - & - & 一 & - & - & - & - & - \\
\hline
\end{tabular}

Table 2 Detection limits/detection ranges of detection methods for microorganisms

\begin{tabular}{|c|c|c|c|c|}
\hline FLISA & S. typhimurium & $3 \times 10^{3} \mathrm{CFU} \mathrm{mL}^{-1}$ & $4 \mathrm{~h}$ & Study support (ESI 5) \\
\hline ELISA & S. typhimurium & $10^{3}-10^{4} \mathrm{CFU} \mathrm{mL} \mathrm{m}^{-1}$ & $>1 \mathrm{~d}$ & Wilanee et al. $2011^{17}$ \\
\hline PCR & S. typhimurium & $10^{2} \mathrm{CFU} \mathrm{mL} \mathrm{m}^{-1}$ & $12-18 \mathrm{~h}$ & Wang et al. $2018^{20}$ \\
\hline ATP bioluminescence & Salmonella & $10^{3} \mathrm{CFU} \mathrm{mL}^{-1}$ & $20 \mathrm{~min}$ & Lee et al. $2017^{22}$ \\
\hline Impedance method & S. typhimurium & $7.6 \times 10^{1} \mathrm{CFU} \mathrm{mL} \mathrm{m}^{-1}$ & $1 \mathrm{~h}$ & Settu et al. $2015^{16}$ \\
\hline
\end{tabular}

after suitable dilution (ESI $6 \dagger$ ). It indicates that the FLISA method has a wide range of applications. Finally, immunofluorescence assay kit method based on doped QDs has a better stability than fluorescent immunochromatographic test strips. When the reagents of FLISA were stored for about 3 months, the LOD of $D$. tsuruhatensis CM13 could still be up to $10^{5} \mathrm{CFU} \mathrm{mL}^{-1}$.

\section{Conclusion}

In this study, stable and high PLQY CdTe : Zn/ZnS novel doped QDs were prepared by water synthesis method. D. tsuruhatensis CM13 extracted from foods were used as the research objects to establish a fluorescence immunoassay (FLISA), thus providing a new detection method for the rapid detection of microorganisms. The whole detection time was less than that of ELISA and this method would be a new protocol for the detection of microorganisms.

\section{Conflicts of interest}

There are no conflicts to declare.

\section{Acknowledgements}

This research is supported by the Fundamental Research Funds for the Central Universities (Program No. 2662019PY067).

\section{References}

1 J. H. Bang and P. V. Kamat, ACS Nano, 2009, 3, 1467-1476.

2 C. Shi, X. Y. Huang, C. Q. Dong, H. J. Chen and J. C. Ren, Chin. Chem. Lett., 2009, 20, 1119-1122.

3 Y. Li, R. Afrasiabi, F. Fathi, N. Wang, C. Xiang, R. Love, Z. She and H.-B. Kraatz, Biosens. Bioelectron., 2014, 58, 193-199.
4 O. Minikh, M. Tolba, L. Brovko and M. Griffiths, J. Microbiol. Methods, 2010, 82, 177-183.

5 Z. Yu, H. Lv and D. Tang, Mater. Lett., 2018, 210, 207-210.

6 J. Yu, J. Su, J. Zhang, X. Wei and A. Guo, RSC Adv., 2017, 7, 17819-17823.

7 A. Yang, Y. Zheng, C. Long, H. Chen, B. Liu, X. Li, J. Yuan and F. Cheng, Food Chem., 2014, 150, 73-79.

8 J. Zhang, S.-Q. Zhao, K. Zhang and J.-Q. Zhou, Chemosphere, 2014, 95, 105-110.

9 N. Gaponik, in Cadmium telluride quantum dots: advances and applications, CRC Press, Boca Raton, Florida, 2013, pp. 6186.

$10 \mathrm{~J}$. Donegan and Y. Rakovich, Cadmium telluride quantum dots: advances and applications, Pan Stanford, 2016.

11 A. Samanta, Z. Deng and Y. Liu, Langmuir, 2012, 28, 82058215.

12 F. Di Nardo, L. Anfossi, C. Giovannoli, C. Passini, V. V. Goftman, I. Y. Goryacheva and C. Baggiani, Talanta, 2016, 150, 463-468.

13 Z. Zhang, X. Xia, X. Xiang, F. Huang and L. Han, Sens. Actuators, B, 2018, 257, 1-8.

14 S. Xiong, Y. Zhou, X. Huang, R. Yu, W. Lai and Y. Xiong, Anal. Chim. Acta, 2017, 972, 94-101.

15 C. Yang, G. Feng, S. Wang, J. Dai, Y. Zhang and S. Zhou, Chem. Phys. Lett., 2017, 684, 409-413.

16 K. Settu, C.-J. Chen, J.-T. Liu, C.-L. Chen and J.-Z. Tsai, Biosens. Bioelectron., 2015, 66, 244-250.

17 W. Chunglok, D. K. Wuragil, S. Oaew, M. Somasundrum and W. Surareungchai, Biosens. Bioelectron., 2011, 26, 3584-3589.

18 S. Xia, Z. Yu, D. Liu, C. Xu and W. Lai, Food Control, 2016, 59, 507-512.

19 Z. Baoqing, L. Taobo, Z. Zhongxu, L. Rui, L. Fan and X. Hengyi, J. Dairy Sci., 2017, 100, 8804-8813. 
20 M. Wang, J. Yang, Z. Gai, S. Huo, J. Zhu, J. Li, R. Wang, S. Xing, G. Shi and F. Shi, Int. J. Food Microbiol., 2018, 266, 251-256.

21 D.-B. Wang, B. Tian, Z.-P. Zhang, J.-Y. Deng, Z.-Q. Cui, R.-F. Yang, X.-Y. Wang, H.-P. Wei and X.-E. Zhang, Biosens. Bioelectron., 2013, 42, 661-667.
22 J. Lee, C. Park, Y. Kim and S. Park, BioChip J., 2017, 11, 287293.

23 T. Wen, R. Wang, A. Sotero and Y. Li, Sensors, 2017, 17, 1973. 24 I. Palchetti and M. Mascini, Anal. Bioanal. Chem., 2008, 391, 455-471. 